\title{
Axial compressive strength determination of hot rolled circular hollow steel sections with initial crookedness between $1 / 2,000$ and $1 / 500$ of the length
}

\author{
Lucas Figueiredo Grilo ${ }^{1 *}$, Ricardo Hallal Fakury ${ }^{1}$, Ana Lydia Reis de Castro e Silva ${ }^{1}$ \\ and Victor Pereira Daldegan ${ }^{1}$ \\ ${ }^{1}$ Department of Structural Engineering, Federal University of Minas Gerais \\ Belo Horizonte, Minas Gerais, 31270-901, Brazil - lucasfgrilo@gmail.com, \\ fakury@dees.ufmg.br, lydia@dees.ufmg.br, \\ victorpereiradaldegan@hotmail.com
}

\begin{abstract}
In this paper, a simple formulation for current application for the axial compressive strength determination of hot rolled circular hollow steel sections, with sinusoidal form initial crookedness and values of the central deflection between $1 / 2,000$ and 1/500 of the length, is proposed. In order to validate the analysis, several numerical models were generated by finite element method, considering members with $1 / 1,000$ of the length initial crookedness, and the results were compared with the values obtained by EN 1993-1-1:2005. Using the methodology of modeling validated, many other members with initial crookedness of 1/2,000,1/1,500,1/750 e $1 / 500$ of the length were analyzed, allowing to obtain the values of the reduction factor, $\chi$, as a function of the reduced slenderness parameter, $\bar{\lambda}$. The values of $\chi$ of members with initial crookedness between these two limits were evaluated by polynomial interpolation.
\end{abstract}

Keywords: steel structures, circular hollow sections, axial compressive strength, initial crookedness, buckling.

\section{Introduction}

The initial geometric imperfections of steel members are inherent to the fabrication process and can be amplified during transportation and assembly of the structure. The value of this imperfection used in the generation of the axial compressive strength curves by European design specification - EN 1993-1-1:2005 - is bellow the tolerance values prescribed by the manufacturing specifications (CEN, 2005; Beedle, 1991; Galambos, 1998). This difference awakes a concern about the actual value of the member's strength when compared to that evaluated according the design specification (Bild \& Trahair, 1989).

* Corresponding author 
The limit for the initial crookedness in the manufacturing specifications for hot rolled circular hollow sections (CHS) is $L / 500$, were $L$ is the length of the member (CEN, 2006; ASTM A501:2005), while the initial crookedness adopted by EN 1993-1-1:2005 is $L / 1,000$ (CEN, 2005; Beer e Shoulz, 1970).

In real situations, it may be necessary to use members with initial crookedness higher than $L / 1,000$. In these conditions, the axial compressive strength of the member is smaller than the value obtained using the European Specification (CEN, 2005). In addition, when the initial crookedness of the members is under $L / 1,000$, it is also interesting to know that the strength then is higher than the specification value. In both cases, the determination of the compressive strength demands experimental and computational procedures.

In this paper, an analytical procedure for the axial compressive strength determination of hot rolled circular hollow steel sections, with initial crookedness between 1/2,000 and $1 / 500$ of the length, is presented. In order to validate the analysis, initially, several numerical models were generated by FEM, using software ABAQUS 6.13 (Hibbit et al., 2007), considering members with $1 / 1,000$ of the length initial crookedness, and the results were compared with the values obtained by the European Procedure. Then, the study was extended to values of initial crookedness of 1/2,000,1/1,500,1/750 and $1 / 500$ of the length, and applied to sections that are not subjected do local buckling.

\section{Behavior of compression members with initial crookedness}

Steel tends to have a perfectly plastic behavior when the internal stresses reach the yield stress. In the case of compression members with initial crookedness, subjected to compression and/or flexural stresses, the yielding of the central section begins in the concave face in the most external fiber of the section (where the stresses are higher) when the compression force starts. With the increase of the force and the displacements, the internal bending moment raises and the yielding spread to the interior of the section, reaching the neighborhood sections (Figure 1-a). After that, the yielding propagates to the convex face (Figure 1-b) and, as the internal stresses continue to raise, the yielding region of the member grows until all the central section yields, 
forming a plastic hinge, as shown in Figure 1-c. In this situation, the member becomes an instable plastic mechanism and collapses (Chen \& Han, 2007).

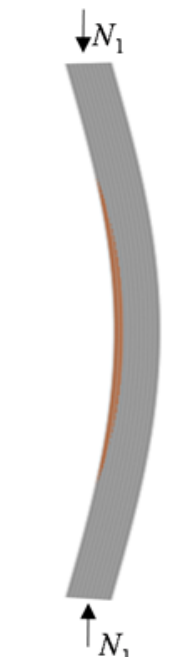

(a)

Beginning of yielding

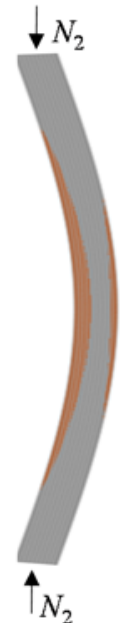

(b)

Yielding propagation

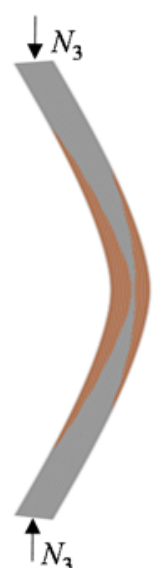

(c) formation

Figure 1 - Collapse of axial compression members with initial crookedness

The force corresponding to the instability is assumed as the axial compressive strength of the member. This strength is inversely related to the initial crookedness and affected by the magnitude and distribution of the residual stresses in the cross section. For higher values of residual stresses, with the occurrence of compression in the concave face (where the yield begins), the loss of stiffness occurs for a smaller compressive force, leading to a smaller axial compressive strength. Otherwise, the loss of stiffness manifests later leading to a higher strength.

In hot rolled sections, the residual stresses are the result of the cooling of the steel, from the lamination to the ambient temperature, occurring non-uniformly in the cross section. During the cooling, the steel reduces volumetrically but presents an increase in its strength and stiffness. This volumetric reduction happens in the parts of the cross section that cool first. These parts stiffen and start to resist to the contraction of the other parts. When the cooling of the section is completed, the parts that cooled first present compressive residual stresses and the others present tension residual stresses. The result of this phenomenon is a gradient of self-equilibrated stresses in the cross section that varies from compression to tension. 
For hollow circular sections, the cooling process of the cross section is characterized by the variation from the external face to the internal face during a short period of time (with compressive residual stresses in the external face and tension residual stresses in the internal face). Therefore, the residual stresses are linearly distributed along the thickness and there is no temperature variation along the circumference of the cross section, with minor values than the values of residual stresses found in open cross sections, such as I, $\mathrm{U}$ and $\mathrm{L}$ profiles.

According to European Convention for Constructional Steelwork (ECCS, 1976), members with hot rolled circular hollow sections are not significantly affected by residual stresses. In these sections, there is a maximum linear variation of $-15 \%$ to $+15 \%$ of the yield strength, as shown in Figure 2. Nowadays these values are still considered acceptable (Grilo, 2015). Recently, Law \& Gardner (2012) obtained the residual stresses in a similar profile, with elliptical cross section, and concluded that these stresses correspond to 10 to $15 \%$ of the steel yield strength, confirming the previous conclusions about the circular hollow sections.

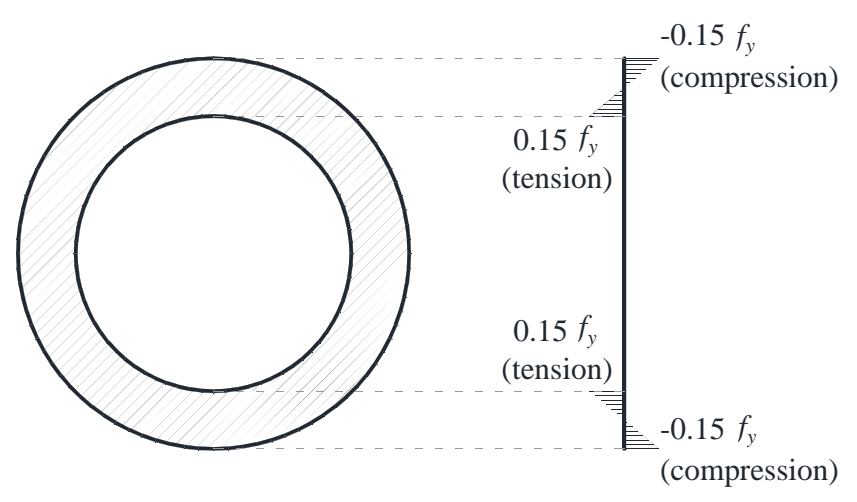

Figure 2 - Residual stresses in hot rolled tubular sections

The form of the curve of compressive force $(N)$ versus deflection $\left(v_{c}\right)$ of the member with initial crookedness is shown in Figure 3. 


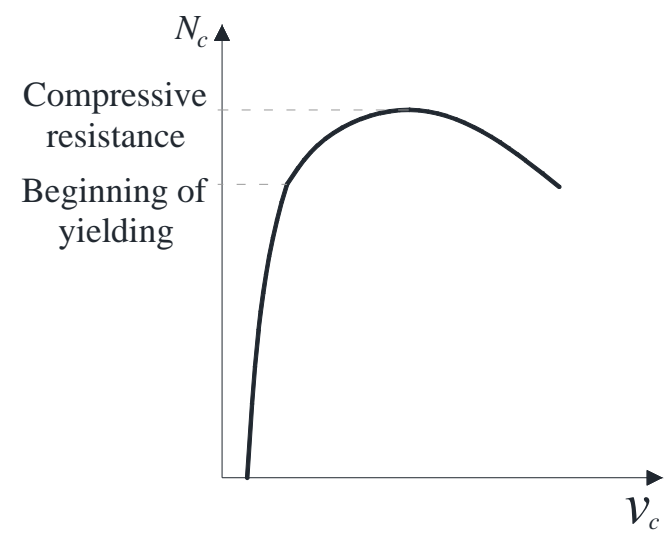

Figure 3 - Curve of forces versus deflection

\section{Resistance force according to EN 1993-1-1:2005}

For the determination of the characteristic axial compressive resistance force of class 1 , 2 and 3 cross-sections, EN 1993-1-1 provides the following expression (CEN, 2005):

$$
N_{b, R k}=\chi A f_{y}
$$

where $\chi$ is a reduction factor related to the compressive strength, $A$ is the gross cross section and $f_{y}$ is the yield strength of the steel. The effects of the initial crookedness are considered in the determination of the factor $\chi$, that is given by:

$$
\chi=\frac{1}{\Phi+\sqrt{\Phi^{2}-\bar{\lambda}^{2}}} \leq 1
$$

In this expression, $\bar{\lambda}$ is the non dimensional slenderness, defined as:

$$
\bar{\lambda}=\sqrt{\frac{A f_{y}}{N_{c r}}}
$$

where $N_{c r}$ is the elastic critical force, and, for the resistance curve " $a$ ", that includes tubular circular profiles, $\Phi$ is given by:

$$
\Phi=0.5\left[1+0.21(\bar{\lambda}-0.2)+\bar{\lambda}^{2}\right]
$$

The expression for $\chi$ adopted by EN 1993-1-1:2005 considers an initial curvature of $L / 1,000$, as stated before. In this expression, adjusted by experimental and numerical analysis, the effects due to residual stresses are also included. 


\section{Development and validation of the numerical models}

\subsection{Introduction}

Numerical models of tubes with initial crookedness subject to axial compression were analyzed and compared with the values obtained using the procedures of the EN 19931-1:2005, presented on Item 3. The numerical analyses were performed using ABAQUS 6.13 , considering different types of elements, meshes and boundary conditions, in order to adjust these analysis parameters (CEN, 2005, Hibbit et al., 2005).

For the determination of the inelastic resistance force it was chosen in ABAQUS the modified Riks method (arc length method), that allows to obtain static equilibrium states during the unstable phase of the structure (Castro e Silva, 2006).

\subsection{Element}

In order to choose the most appropriate element for the models it was tested six different elements: S4, S4R and S8R (shell elements) and C3D8, C3D8R and C3D20R (solid elements). In each group, the last two consider reduced integration.

It was observed that the models with solid elements need a very refined mesh to achieve the same results of the shell elements of the same order and that they have a bigger computational cost, therefore, they were abandoned.

Among the shell elements, the quadratic element S8R, with reduced integration, presented better results, adapting perfectly to the geometry of the problem and requesting a coarse mesh to obtain the same results of the linear elements S4 and S4R. Besides, the fact that this element may have curve sides is an advantage for circular tubes because it makes the modeled geometry exact and not approximated by segments of line.

The S8R element has eight nodes (Figure 4-a), with four integration points on the surface (Figure 4-b), and was configured to use five integration points along its thickness (Figure 4-c). These points are necessary to represent the nonlinear behavior of the strains and stresses. For the numerical integration, it was considered the Simpson rule. Each node has six degrees of freedom (three translations and three rotations), which are associated to the reference surface (Hibbit et al., 2005). 


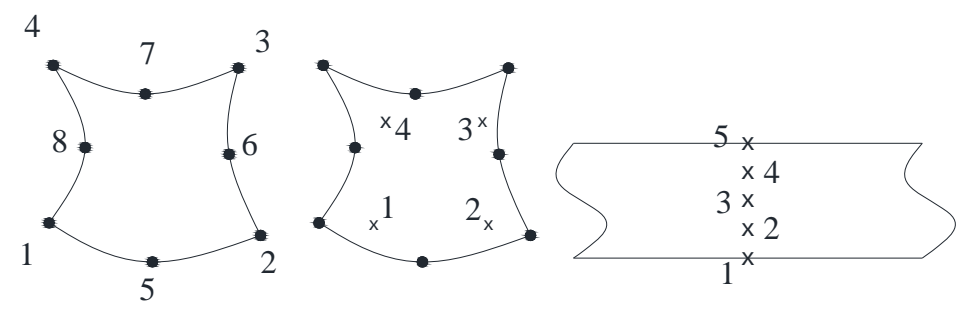

(a)

(b)

Nodes

Surface integration points

(c)

Integration points along thickness

Figure 4-Shell element S8R

\subsection{Modeling}

The tubes models were generated with initial crookedness, taken as $L / 1,000$, the same value adopted on the procedure of EN 1993-1-1:2005. It was considered an elasticplastic bilinear stress-strain diagram for steel. The yield strength of the steel was taken as $355 \mathrm{MPa}$.

The residual stresses were neglected in the numerical models since its variations occurs only along the thickness and has an insignificant effect on the global behavior of the structure.

It was modeled one quarter of the tube, as shown in Figure 5, taking advantage of the longitudinal symmetry plane, that goes through a principal centroidal axis of the crosssection, and a transversal plane, on the half of the length.

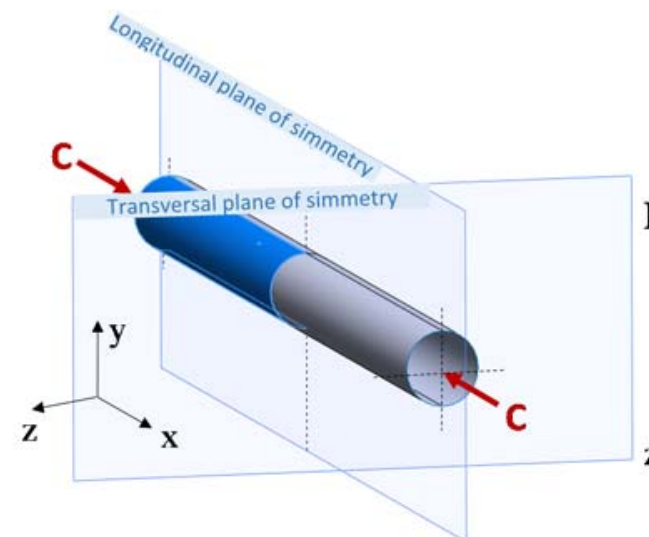

(a) Symmetric planes

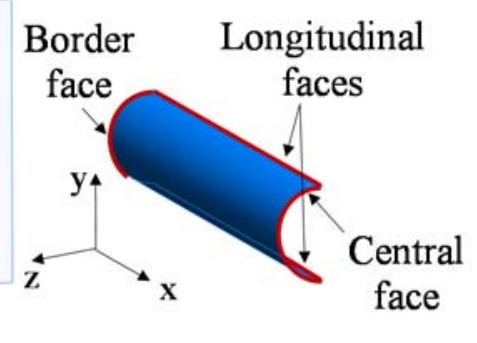

(b) Model faces

Figure 5 - Symmetry planes considered 
To apply constrains and the boundary conditions, the border face (Figure 5-b) was totally connected with a fictional point located on the geometric center of the cross-section of the tube. In this point, the translations in the $y$ and $z$ directions and the rotations on $x$ and $y$ were restrained. The nodes of the longitudinal faces were restrained to translations on the $z$ direction and to rotations on $x$ and $y$. The nodes of the central face were restrained to translations on the $\mathrm{x}$ and $\mathrm{z}$ directions and to rotations on $\mathrm{y}$ and $\mathrm{z}$.

The compressive force was applied on the created fictional point and ABAQUS was configured to not alter the direction of the force with the nodes orientation change trough the analysis.

\subsection{Mesh sensitivity test}

In order to find the best discretization of the models, a sensibility test of the mesh for S8R element was made by the variation in the quantity of elements in the perimeter. The circular hollow sections: CHS $73 \times 3.6$ (diameter/thickness ratio, $d / t$, equal to $73 / 3.6$, i.e. 20) and CHS $323.8 \times 6.4(d / t$ equal to 50$)$, with slenderness parameters approximately 0.80 and 1.60, respectively, were considered. In Figure 6, it can be seen the convergence results of the mesh sensibility test.

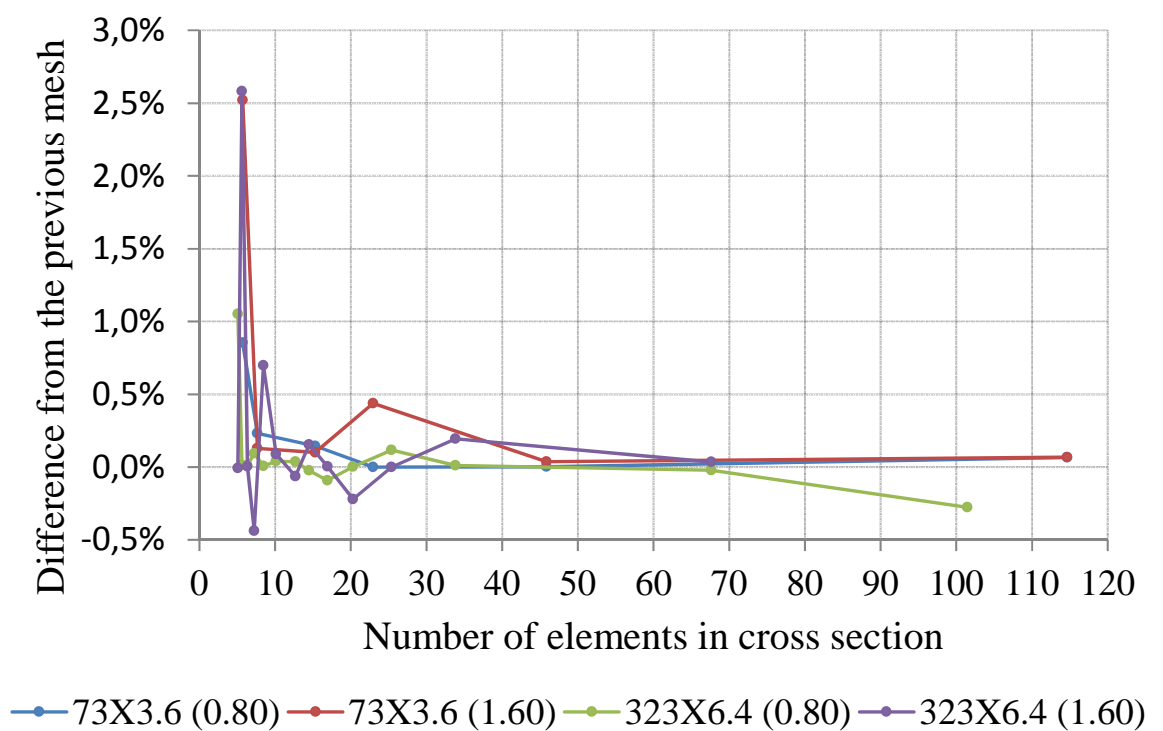

Figure 6 - Mesh sensibility test 
The figure shows that for a number of elements in the cross section higher than 10 , the difference between the results is negligible for all models tested. Therefore, aiming to reduce the error in the modeling to a minimum and optimizing the processing time, it was adopted 15 elements in the cross section in this work.

\subsection{Results}

The circular hollow sections: CHS $73.0 \times 7.1$, CHS $73.0 \times 3.6$ and CHS $323.8 \times 6.4$, with $d / t$ equal to $10.3,20.3$ and 50.6, respectively, were considered. Twenty models of each of the three chosen cross sections were analyzed considering the variation in the reduced slenderness parameter $(\bar{\lambda})$ from 0.3 to 2.6 , with increments of approximately 0.133 . The superior limit 2.6 corresponds to maximum value of the slenderness $(\bar{\lambda})$ that is allowed by EN 1993-1-1:2005 for axial compressive members made of steel with yield strength of $355 \mathrm{MPa}$.

In Figure 7 it can be observed the yielding spreading with the raise of the transversal displacement.

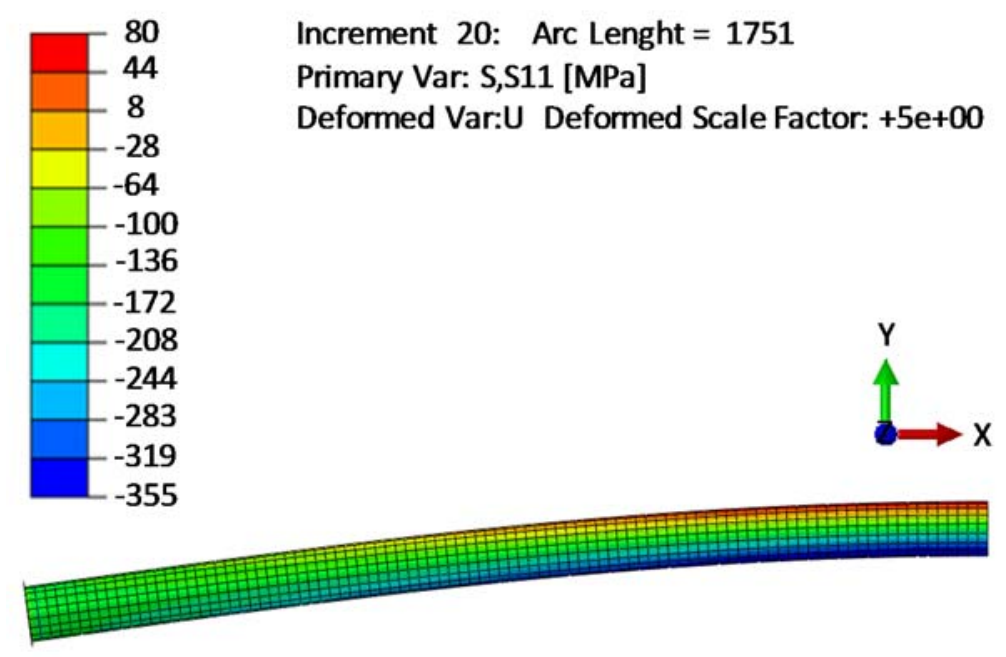

Figure 7-Partial yielding of the central cross section

The curves of $\chi$ versus $\bar{\lambda}$ for the three cross sections were obtained generating the plots of the differences between these curves and the curves of EN 1993-1-1:2005, shown in Figure 8. 


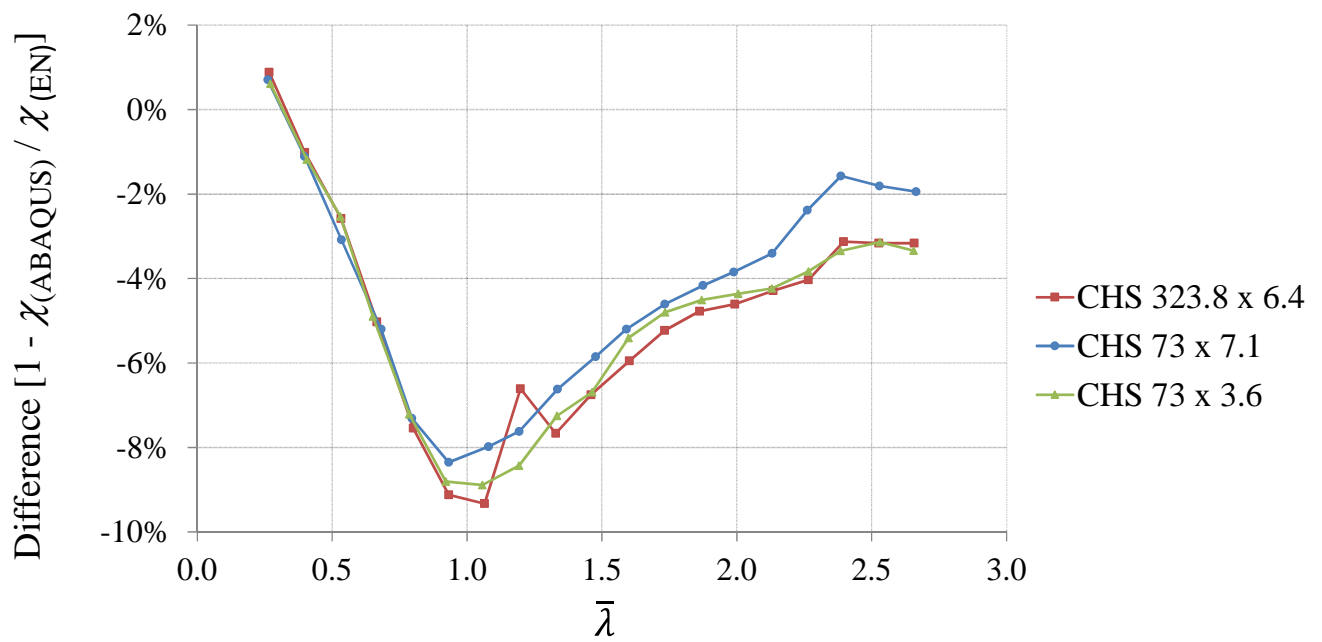

Figure 8 - Difference between the curves of $\chi$ versus $\bar{\lambda}$ of Abaqus and of EN 1993-1-1

The maximum difference observed between the numerical results and the results evaluated using EN 1993-1-1 was under 9.3\%. Therefore, the numerical modeling parameters were considered satisfactory for this study.

\section{Analysis of models with various initial crookedness}

\subsection{Considerations}

Using the same numerical modeling approach validated for initial crookedness of $L / 1,000$, it were analyzed members with initial crookedness of $L / 2,000, L / 1,500, L / 1,000$, $L / 750$ and $L / 500$. For each value of the initial crookedness the analysis considered the variation in the length of the tube with $\bar{\lambda}$ in the range of 0.3 to 2.6 , with increments of 0.27. The circular hollow sections analyzed are presented in Table 1.

Table 1 - Circular hollow sections analyzed

\begin{tabular}{lccc}
\hline \multicolumn{1}{c}{ Profile } & $\begin{array}{c}\text { Diameter }(d) \\
\mathrm{mm}\end{array}$ & $\begin{array}{c}\text { Thickness }(t) \\
\mathrm{mm}\end{array}$ & $\mathrm{d} / \mathrm{t}$ \\
\hline 1 (CHS $88.9 \times 3.6)$ & 88.9 & 3.6 & 24.7 \\
2 (CHS $141.3 \times 8.0)$ & 141.3 & 8.0 & 17.7 \\
3 (CHS $141.3 \times 16.0)$ & 141.3 & 16.0 & 8.8 \\
4 (CHS $273.3 \times 6.4)$ & 273.3 & 6.4 & 42.7 \\
5 (CHS $355.6 \times 12.5)$ & 355.6 & 12.5 & 28.4 \\
\hline
\end{tabular}




\subsection{Comparison between models}

The values of $\chi$ as a function of $\bar{\lambda}$, obtained in the numerical analysis, for the circular hollow sections presented in Table 1, were compared between each other. The mean values and the standard deviations obtained are presented in figures 9 and 10, for each considered value of initial crookedness.

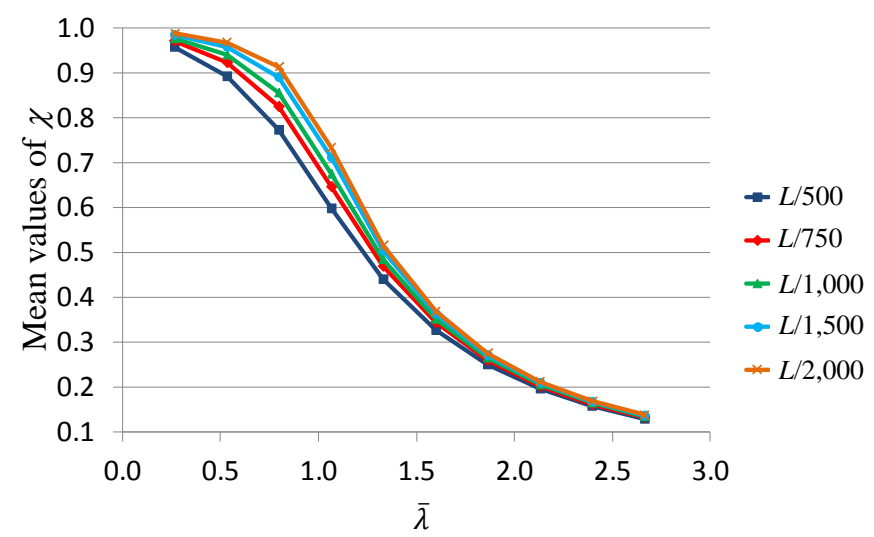

Figure $9-$ Mean values of $\chi$ obtained with the numerical models

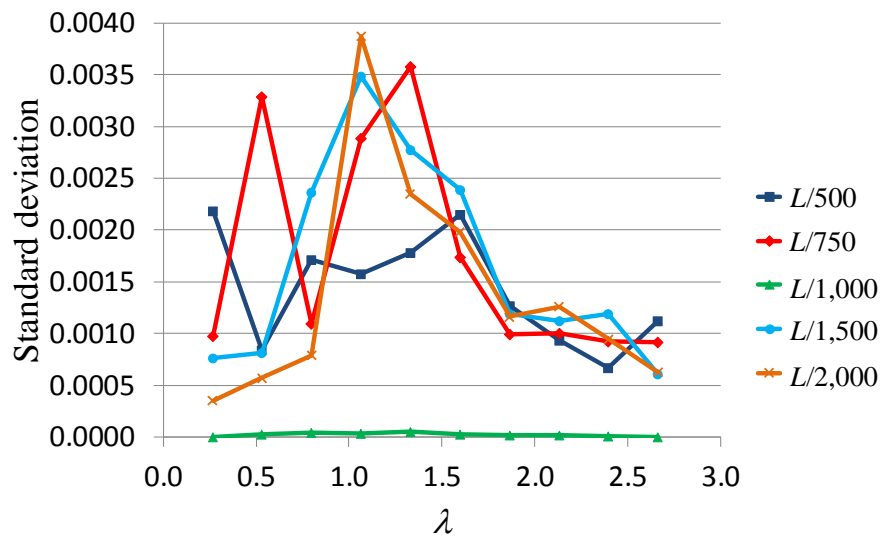

Figure 10 - Standard deviation of $\chi$, obtained with the numerical models

The percental differences observed for the values of $\chi$ for all models were less than 2.5\%. Theoretically, once the compared models are similar, only varying the cross sections, the values of $\chi$ for models 1 to 5 should be the same. The small variation observed probably is related to numerical modeling approaches and was considered satisfactory for this study.

\section{Formulation proposed to evaluate $\chi$}

In order to propose a formulation to evaluate $\chi$ as a function of $\bar{\lambda}$, the numerical results of profile 1, from Table 1, were parameterized and compared with the other profiles 
results.,. As a start point, the results of members with minimum and maximum initial crookedness $(L / 2,000$ and $L / 500$ ) were used and polynomial adjustments were made for each one of the curves. In these adjustments, each curve was represented by 3 polynomials, with breaking points determined to improve the polynomial fitting, where the reduced slenderness parameter $(\bar{\lambda})$ is equal to 1.07 and 1.60 (Figure 11 ), as follows:

- for $0<\bar{\lambda} \leq 1.07$

$f_{1 / 500}(\bar{\lambda})=0.225 x^{4}-0.624 x^{3}+0.218 x^{2}-0.172 x+1$
$f_{1 / 2.000}(\bar{\lambda})=-0.543 x^{4}+0.624 x^{3}-0.2815 x^{2}-0.004 x+1$

- for $1.07<\bar{\lambda} \leq 1.60$

$f_{1 / 500}(\bar{\lambda})=0.3203 x^{2}-1.3658 x+1.6912$

$f_{1 / 2.000}(\bar{\lambda})=0.4802 x^{2}-1.95965 x+2.2744$

- for $1.60<\bar{\lambda} \leq 2.66$

$f_{1 / 500}(\bar{\lambda})=-0.05382 x^{3}+0.45904 x^{2}-1.39295 x+1.5999$
$f_{1 / 2.000}(\bar{\lambda})=-0.07449 x^{3}+0.6230 x^{2}-1.83665 x+2.0172$

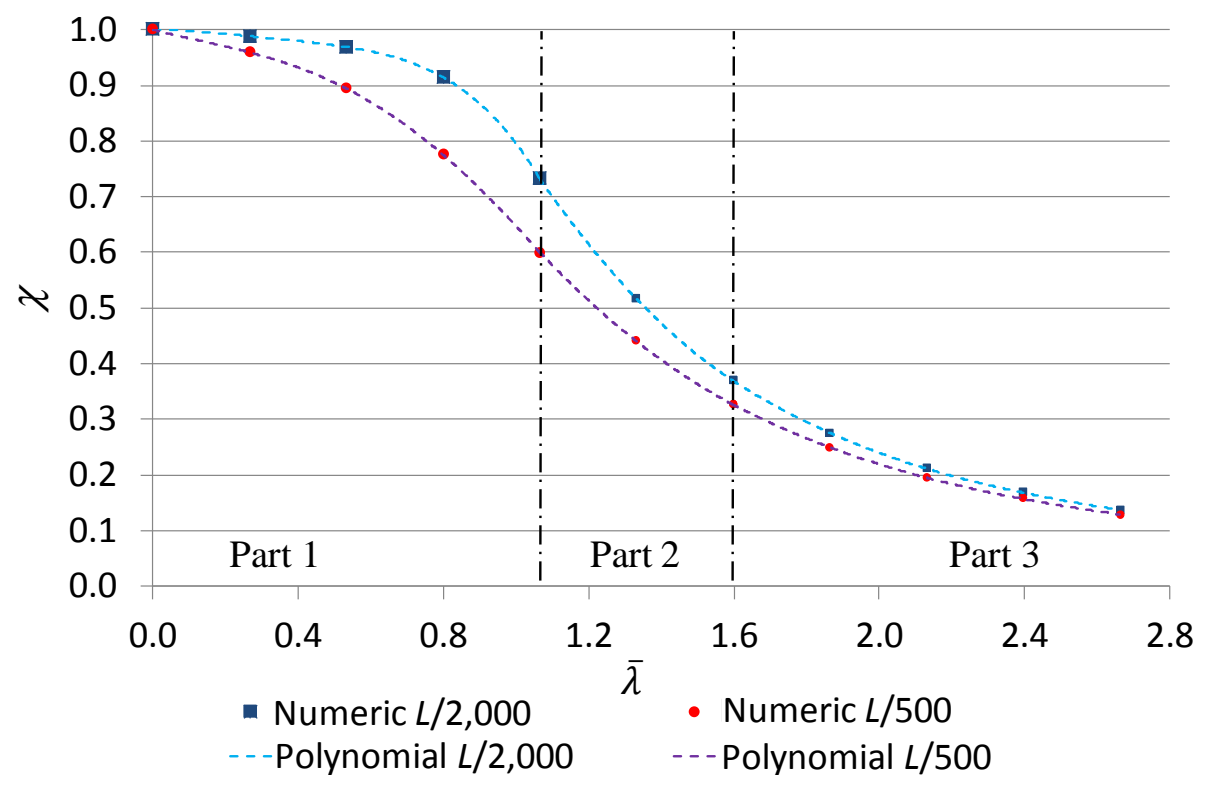

Figure 11 - Polynomial adjustments for initial crookedness of $L / 2,000$ and $L / 500$ 
As can be seen in Figure 11, for each adjustment was obtained results for values of $\bar{\lambda}$ between 0 and 2.66. After that, the procedure of Munoz-Rojas and Kuhl (2011) for interpolation of generic polynomial curves was adopted so as any curvature between $L / 2,000$ and $L / 500$ could be used, leading to:

$$
f_{1 / \delta}(\bar{\lambda})=f_{1 / 500}(\bar{\lambda})+\left[\frac{(1 / \delta-1 / 500)}{(1 / 2,000-1 / 500)}\right]\left[f_{1 / 2,000}(\bar{\lambda})-f_{1 / 500}(\bar{\lambda})\right]
$$

where $f_{1 / \delta}(\bar{\lambda})$ is the equation of the curve $\chi$ versus $\bar{\lambda}$ for the member with the desired relative initial crookedness $(1 / \delta)$ and $f_{1 / 2.000}(\bar{\lambda})$ and $f_{1 / 500}(\bar{\lambda})$ are the polynomial equations of the initial crookedness of $L / 2,000$ and $L / 500$, respectively. The comparison between the proposed formulation, with initial crookedness equals to $L / 1.000$, and the EN 1993-1-1:2005 formulation is shown in Figure 12.

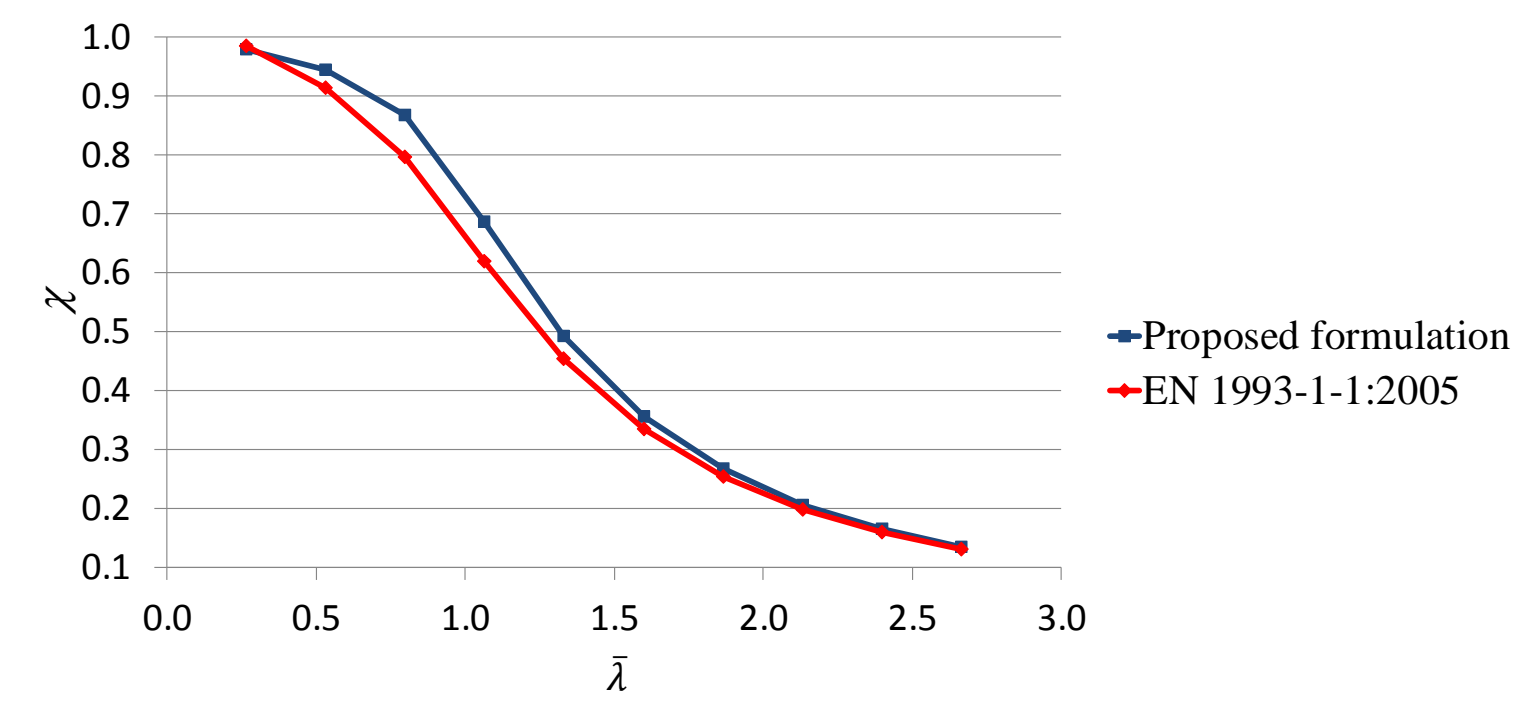

Figure 12 - Comparison between the proposed formulation and EN 1993-1-1:2005 for initial crookedness equals to $L / 1,000$

The difference of the results between the proposed formulation and EN 1993-1-1:2005 are below $10 \%$, as expected, once the numerical model was calibrated with the European Standard (Figure 8).

In order to check the proposed formulation to evaluate $\chi$, given by Equation (6.7), and compare the values of $\chi$ for each $\bar{\lambda}$, the curves for initial crookedness equals to the ones simulated numerically $(L / 2,000, L / 1,500, L / 1,000, L / 750$ and $L / 500)$ were generated. The obtained results are presented in Figure 13, as a percental difference, for each profile (see Table 1). 


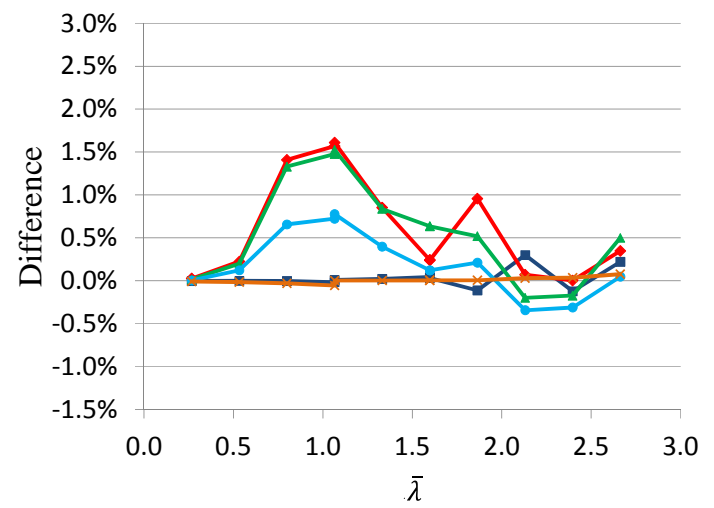

(a) Profile 1 (CHS $88.9 \times 9.3$ )

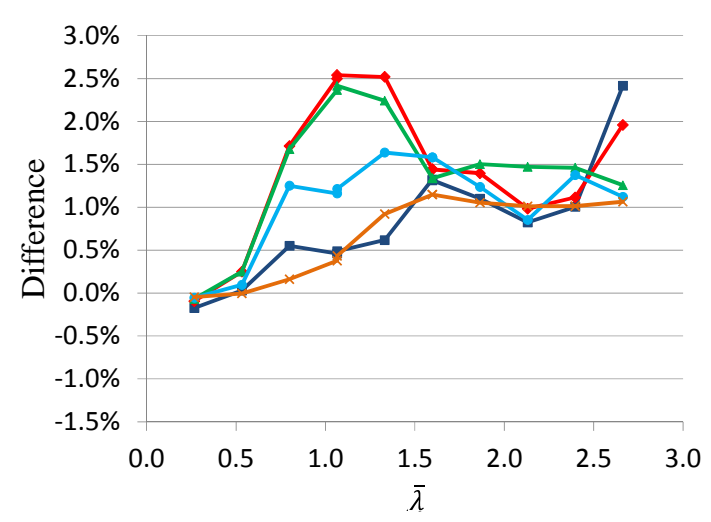

(c) Profile 3 (CHS $141.3 \times 16.0$ )

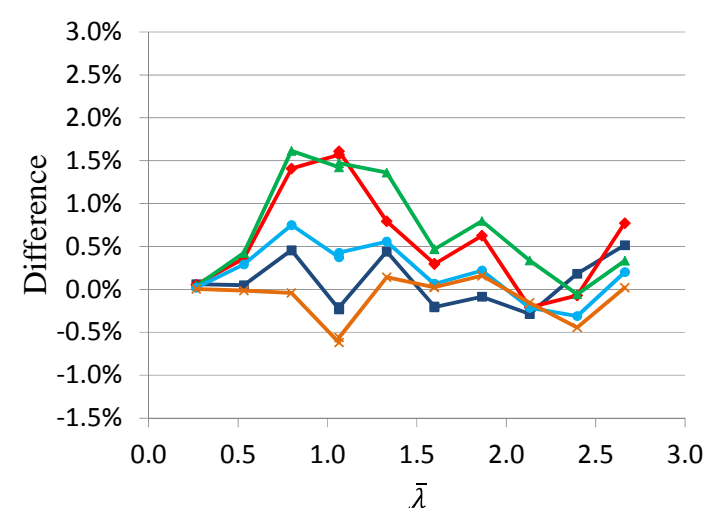

(e) Profile 5 (CHS $355.6 \times 12.5$ )

$$
-L / 500 \rightarrow L / 750 \quad-L / 1.000 \rightarrow L / 1.500 * L / 2.000
$$

Figure 13 - Percental differences between numerical results and those obtained by Equation (6.7)

As can be seen, the biggest percental difference in the value of $\chi$, when comparing the values obtained by the proposed formulation and the numerical models, was of $2.5 \%$, that can be considered a good agreement and adequate for structural design purpose. 


\section{Conclusions}

This work proposes a formulation to evaluate the factor $\chi$ of members with hot rolled circular hollow steel sections, with initial crookedness between $L / 2,000$ and $L / 500$, and consequently its axial compressive strength, using Equation (3.1).

In order to obtain the presented formulation, a numerical analysis was performed and checked with the European code EN 1993-1-1:2005, which considers an initial crookedness of $L / 1,000$. After that, models with initial crookedness of $L / 2,000, L / 1,500$, $L / 750$ and $L / 500$ were generated, and their curves of $\chi$ versus $\bar{\lambda}$ were plotted, making a polynomial adjustment with 3 segments per curve.

The expressions obtained for the initial crookedness of $L / 2,000$ and $L / 500$ were polynomially interpolated, leading to the proposed formulation for the curve of $\chi$ versus $\bar{\lambda}$ of members with any initial crookedness between these two limits.

The results evaluated with the proposed formulation were checked with the numerical results obtained with ABAQUS, and they were considered acceptable, presenting differences under $2.5 \%$ between them.

Finally, the formulation proposed is polynomial, therefor it can be easily computationally implemented, allowing to determine the factor $\chi$ and, consequently, to evaluate the axial compressive strength of members with hot rolled circular hollow sections with different initial crookedness by Equation (3.1).

\section{Acknowledgments}

The authors acknowledgment the support of the Brazilian research agencies CNPq, CAPES and FAPEMIG.

\section{References}

ASTM - Standard specification for hot-formed welded and seamless carbon steel structural tubing, ASTM A501, West Conshohocken, 2005.

BEEDLE, L. S. Stability of Metal Structures: A World View, Structural Stability Research Council, Lehigh University, 2nd Ed., Bethlehem, Pa, 1991.

BILD, S.; TRAHAIR, N. S. In-Plane Strengths of Steel Columns and Beam-Columns. Journal of Constructional Steel Research, 13(1), 1-22, 1989. 
GALAMBOS, T. V., Guide to Stability Design Criteria for Metal Structures, 5th Ed. Wiley, New York, 1998.

CEN. EN 1993-1-1 : Design of steel structures - Part 1-1: General Rules and Rules for Buildings, 2005.

CEN. EN 10210-2: Hot finished structural hollow sections of non-alloy and fine grain steelsPart 2: Tolerances, dimensions and sectional properties, CEN, 2006.

BEER, H.; SCHULZ, G. Bases théoriques des courbes européennes de flambement, Construction Métallique, 3, 37-57, 1970.

HIBBIT; KARLSSON; SORENSEN. ABAQUS/ Standard - User's Manual, USA, 2005.

CHEN, W. F.; HAN, D. J.; Plasticity for Structures Engineers, Springer-Verlag, New York, USA, 2007.

European Convention for Constructional Steelwork - Committee 8. Manual on Stability of Steel Structures, ECCS, 2nd Ed. 1976.

GRILO, L. F. Study of the Behavior of Built-Up Members Composed of Concentric Hot Rolled Circular Hollow Sections Under Compression. MSc. Dissertation, Federal University of Minas Gerais, Brazil, 2015 [in Portuguese].

LAW, H. K.; GARDNER, L. Lateral Instability of Elliptical Hollow Section Beams. Engineering Structures, v. 37, 2012.

A. L. CASTRO e SILVA R., Non-linear Numerical Analysis of Local Buckling of Structural Steel Profiles Under Single Axis Compression. PhD Thesis. Federal University of Minas Gerais, Brazil, 2006 [in Portuguese].

P. A. MUÑOZ-ROJAS, P. T. R MENDONÇA, I. L. BENVENUTTI, G. J. CREUS, Modeling nonlinear viscoelastic behavior of High Density Polyethylene (HDPE): application of stress-time equivalence versus interpolation of rheological properties. In III International Symposium on Solid Mechanics. Florianópolis, Brazil, 2011. 